\title{
An Object-Oriented Dialog System for Use in Computer-Aided Teaching
}

\author{
Irene Luque Ruiz, Gonzalo Cerruela García, and Miguel Ángel Gómez-Nieto \\ Department of Computing and Numerical Analysis. University of Córdoba. \\ Campus Universitario de Rabanales, Building Einstein, Plant-3. E-14071 Córdoba (SPAIN) \\ \{mallurui, gcerruela, mangel\}@uco.es
}

\begin{abstract}
In this paper is proposed an ontology based on the object-oriented paradigm for the development of explanatory systems to simulate, in an appropriate way, the professor-student interactions carried out in the teaching processes. The knowledge explained in the dialogue process is represented at different levels of complexity by means of a network of multi-connected units of knowledge. Under the proposed model an open explanatory dialog system is built and incorporated into the Virtual Chemistry Laboratory, a virtual system for practical chemistry learning, developed by the authors.
\end{abstract}

\section{Introduction}

The teaching process is changing in the last years thanks to the use of computers and the development of intelligent tutorial systems. These systems are widely used in fields such as chemistry, where numerous tutorial and learning systems have been developed [1-4]. Nowadays, advances in computing and resources have allowed the development of systems that aim to place the student in a virtual world - the closest thing to a real chemistry lab - in order to carry out experiments that are either considered too dangerous or expensive, or when there is simply a lack of laboratory resources and/or time for large numbers of students $[5,6]$.

The definitive installation of these systems will suppose a drastic change in the current paradigm teaching-learning, although for that is necessary of a technological advance in the communications and software development based on teaching models that simulate, in an appropriate way, the present teaching to the use of the information technologies.

Usefull software that simulates the process of real teaching should keep in mind that professor-student interactions cannot be restricted to the mere presentation of information to the student through help options included in these systems, the access to documents or pages and addresses Web. The professor-student interactions are based, mainly, for doubts or questions that in fact arise to the student when the student tries to assimilate the contents of these kinds of documents, needing to solve some doubts for a correct assimilation of this information. 
Therefore, the teaching-aided systems should have the necessary functionality to allow the student to ask questions, and the system (that simulates the professor) can respond them in an appropriate way, that is to say, depending on the level of the student's knowledge, which is the foundation of the dialog or explanatory systems.

The development of the explanatory systems is very complex. In the first place, it is necessary to define an ontology on which the teaching process is based on the context of the professor-student communication [7-8]. On basis of this ontology, it is necessary to model the knowledge that will be transmitted to the student, and to develop a series of components in order to perform the professor-student communication process, allowing the student to ask questions, and that the professor (the explanatory system) transmits the explanation with a form and content appropriate to the student's knowledge.

In the last decade have been developed a great number of explanatory systems oriented to specific fields of the sciences, engineering and humanities. Mainly, the systems are ad-hoc systems that hardly can be adapted to be used in other areas or educational environments, because are based in an ontology and knowledge model restricted to the educational problem for which its were built. The challenge in next years is the construction of open explanatory systems that can be integrated, with few or any adaptations, in any intelligent tutorial system.

In the present study is proposed an ontology for the development of explanatory systems and a model for the representation of the knowledge that is transmitted in the professor-student interactions. Under this model an open explanatory dialog system has been developed and incorporated into the Virtual Chemistry Laboratory [5], a model previously proposed by the authors. The proposed theoretical model and its development under the object-oriented paradigm are presented, alongside its application to the building of an explanatory system for use in the help feature of a chemistry laboratory teaching application [5].

\section{A Dialog System for the Building of Explanations}

Virtual Chemistry Lab (VCL) [5] is multimedia teaching software aimed at simulating a working environment (in this case a chemistry lab) where students can work in a virtual environment in a similar way to they would in a real practice lab at any teaching center. The help system included in $V C L$, based on a web tutorial, could not be classed as an interactive virtual teacher, rather as a complete textbook available at any moment. With this in mind, development of an interactive dialog system to allow the student to ask questions about any aspect of the lab (materials, working methods, etc.) as well as inquire about theory and/or technique for chemistry experiments would complete the teaching model of the system.

Creation of the dialog system should provide complete representation of the real teaching model; in other words, all the elements of the real educational process involved in the teaching of practical chemistry technique will have their virtual equivalent, as shown in Table 1. 
Table 1. Correspondences between the real world and the $V C L$ virtual world

\begin{tabular}{|llc|}
\hline VCL Virtual World & $\leftrightarrow$ & Real World \\
3D Virtual environment & $\leftrightarrow$ & Chemistry lab \\
On line help system & $\leftrightarrow$ & Text / Reference book \\
$\begin{array}{l}\text { Interactive dialog sys- } \\
\text { tem }\end{array}$ & $\leftrightarrow$ & Lab practice teacher \\
\hline
\end{tabular}

The first objective of the present study was to propose an ontology for the dialog process between the user and the $V C L$, which could be integrated into the $E(V)=M+$ $m$ (Virtual Experiment is represented by means of Materials and a method), paradigm on which it is based [5]. This approach has to take into account the adaptation for the student of the explanatory dialog with respect to how the dialog process is fairing (e.g. using more generic explanations if the student displays, through his questions, a significant lack of knowledge about the topic).

Creation of an interactive dialog system incurs the need to model a complete knowledge network composed of multi-connected nodes, such that it may be browsed using the functions connecting the different network nodes $[8,9,10,11]$.

The proposed model is based on the formulation of suitable paradigms for the representation of the knowledge to be taught, the student model, the content planner (teaching component) and the dialog planner (interface), in line with existing proposals concerning ITS components [12, 13].

Figure 1 shows the architecture of the proposed model in a component diagram [14]. Student and knowledge models are represented by a Knowledge Base that stores the concepts to be taught and their relationships, together with the belief of the student's knowledge of the concepts. The dialog generator is represented by three components: the Dialog Planner, which takes on the role of teacher and handles communication with the student, extracting explanations from the knowledge base to display them in an order determined by the Organizer, whose Content Planner presents the concepts to be explained, based on the knowledge extracted from the knowledge base.

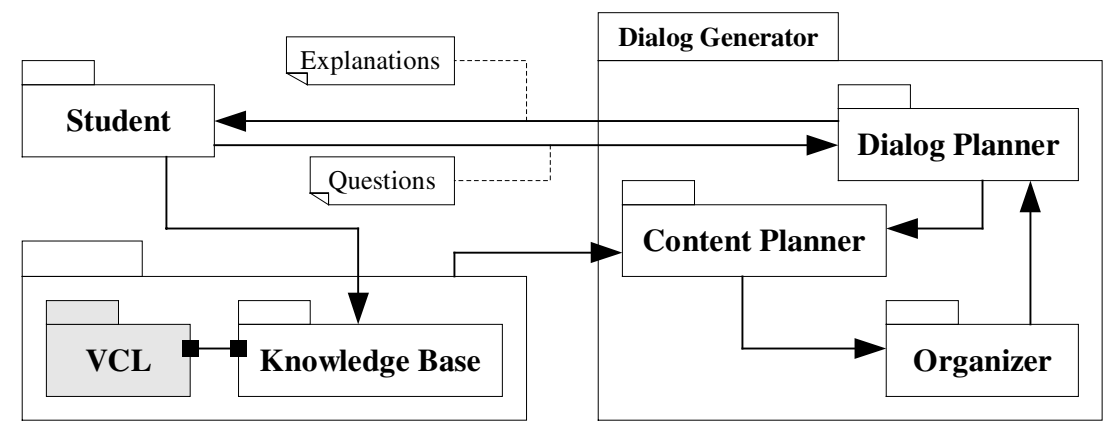

Fig. 1. Component diagram of the proposed model 


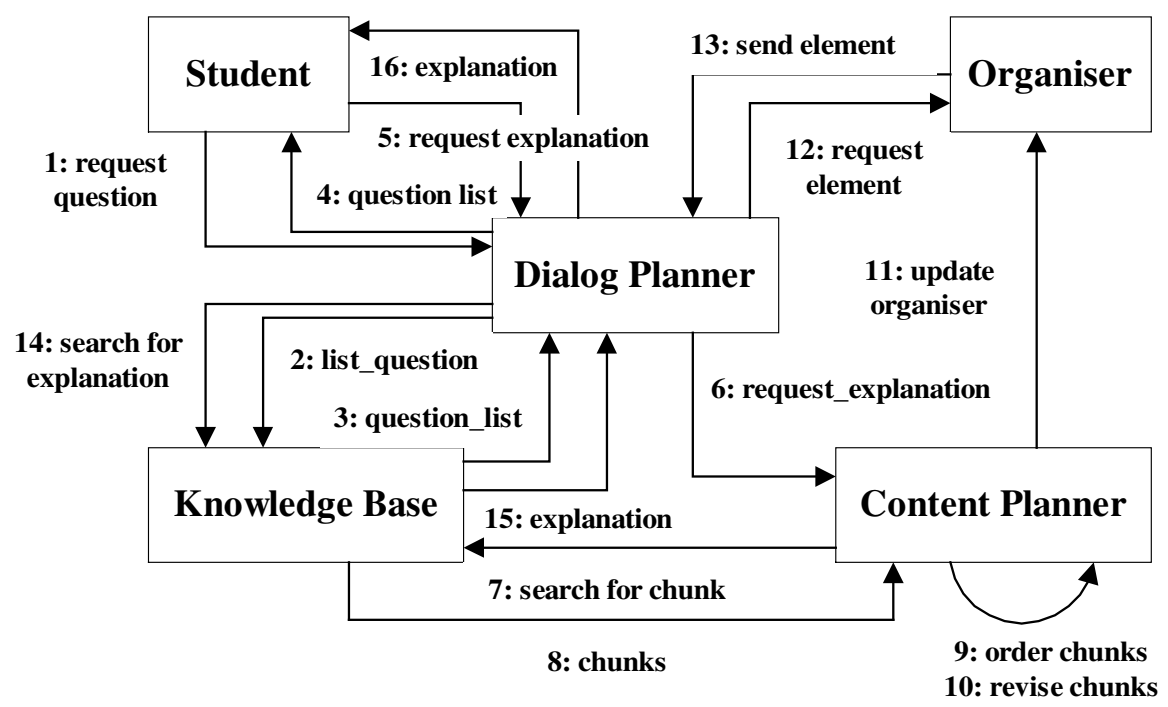

Fig. 2. Explanatory process in the dialog system

As shown in Figure 1, the dialog generator has been integrated with the components of the $V C L$ [5], thus allowing the creation of explanations for any element of the $V C L$ and the incorporation of the explanatory system in its virtual interface.

\subsection{Generating Explanations}

The proposed discourse model is student-dominated [15, 16], where student initiative is limited to asking questions, and is based on the use of explanatory transactions whose aim is to simulate the real-world interaction between students and their lab teacher and allows the dialog flow shown in the collaboration diagram [14] in Figure 2.

A hierarchical model of a dialog structure may produce a useful basis for modeling the organization of interactions, and as an explanation may be expressed as a plan, then a higher level rule for planning transactions will describe an application and will consist of an opening delimiting exchange, a sequence of pedagogical exchanges and a closing delimiting exchange.

In the proposed model, student initiative is limited to asking questions about any material or conceptual element appearing in the experiment to be performed, while the system has the initiative when deciding on the form and content of the explanation.

When an explanation has not been fully understood by the student, the student can either ask the system for clarification or ask new questions. Clarifications on a topic are dealt with thus: (a) Revision of the prerequisites and explanation of the subobjectives which were not explained, (b) The use of another explanatory paradigm, different from its predecessor; or (c) Asking the student questions to reappraise his knowledge level, making a global evaluation of the student through a test examination. 


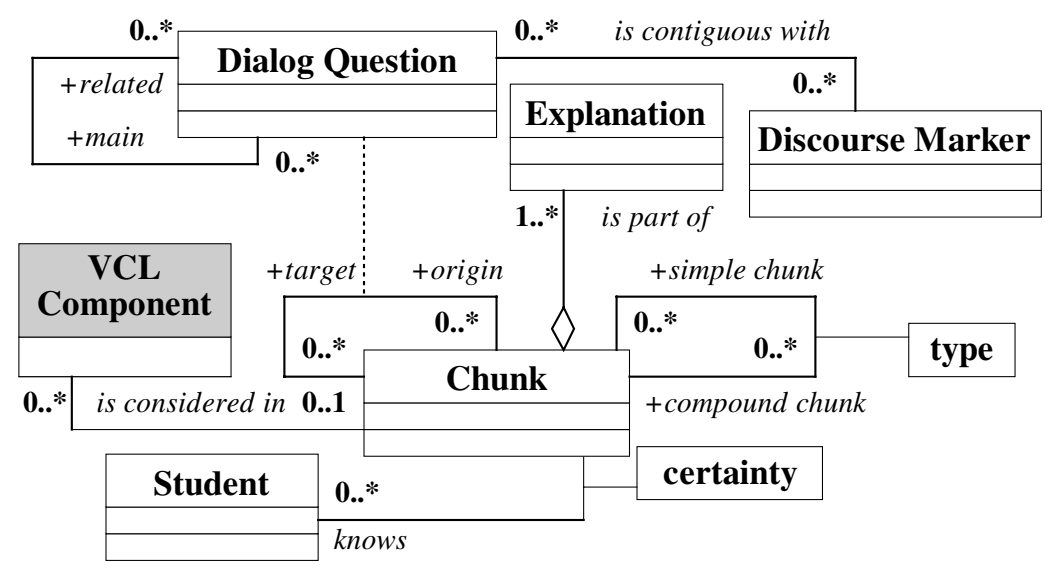

Fig. 3. Class diagram of the proposed model

However, sometimes the student is not really aware of not understanding what is being explained, and it is up to the system to identify the problem and attempt to correct this situation. This happens when the student does not correctly answer the questions posed by the system, and it is more effective to somehow show the student why the answer was wrong instead of simply supplying the correct answer.

To find out where the student was going wrong, the system places in the discourse model the assumptions about the user's knowledge level that had been used to plan the explanation. If the system has assumed that the user had a good knowledge of certain information, but this has proved to be untrue, it may presume that the root of the problem is that the information was unknown to the user (case a), and propose an explanation.

If the system is unable to find a reason for the confusion, it will try to explain the information in another way (case b). In order to achieve this, when there is more than one possible way of explaining something, the discourse model includes links to other forms of explanation.

These clarification subdialogs, which appear as a result of a lack of understanding on the student's part, are dealt with as interruptions and not as part of the prior discourse, so the planned discourse will continue when clarification has been attained.

To add coherence to these clarification subdialogs, there is an interruptions transaction rule responsible for planning appropriate opening and closing sentences for the dialog, which includes the use of discourse markers (Figure 3) that point to the beginning and end of an interruption (e.g. "Anyway" or "Getting back to where we were...".

\subsection{Management Knowledge to Be Taught}

Teaching-aided systems require knowledge to be explained must be represented as related portions for be used depending of the pedagogical features required for each instance [9]. 
Under an object-oriented paradigm (used in this work) the use of frames - represented as classes - allows faithful representation of these related portions of knowledge. Thus, given a discourse domain corresponding to the topic to be taught, the knowledge elements (and their relationships) of which it is comprised may be structured in the form of a network and represented as classes and class associations, as shown in the class diagram [14] in Figure 3.

Knowledge elements have been named chunks, such that a node in the knowledge network may be defined by a name that relates the chunk to its teaching material (explanation).

The relationships (edges between nodes or chunks of the knowledge network) have a specific meaning, an address, and may join pairs of nodes; different types of relationships are allowed, characterized by the Type attribute (Figure 3). This attribute permits represent prelations or prerequisites between chunks (the root node must be known for the target node to be taught), as well as, aggregation or composition relationships between chunks (the root node is a subset of the knowledge in the target node).

Thus, taking a node (knowledge chunk) as a learning objective, all prerequisite relationships arriving at that node may be analyzed, and a check can be made to ensure the student knows each and every node. In this way, if one of the nodes is unknown, it can be marked as a learning objective.

Explanations should be related to the chunk network so that it can be presented to the student when working with the chunk in question. The class diagram in Figure 3 shows how a chunk may contain zero (composite chunks) or several explanations, each of which has a series of distinguishing features and may contain text or graphical information.

Figure 3 also shows a reflexive association of the chunk class, which allows the representation of the directional knowledge (the prerequisites). Additionally, this association is characterized by the Dialog Question class, which in turn maintains a reflexive association with the relationships that exist between specific frames of the knowledge domain.

Representation of student knowledge is based on the knowledge on chunks. Given a certain object from the model (e.g. the titration of $0.1 \mathrm{~N} \mathrm{HCl}$ with $0.1 \mathrm{~N} \mathrm{NaOH}$ ), the user-modeling component works out whether or not the student knows the element.

The extent of the student's awareness of the knowledge elements is represented by certainty measurements, defined by the labels "known", "perhaps known", and "unknown", which characterize the association between the student and the chunk, as shown in Figure 3. These labels provide a simple representation of the degree of belief assigned to the student's knowledge of a concept.

Since the system requires information about the student in order to produce a personalized dialog, the student model is based on examination of the Student class in which contains, among others: a base level of the student knowledge (following the stereotype model [16]), which is update in terms of the history of student-system interaction, and the session level or evaluation of the student performance during a specific session. 


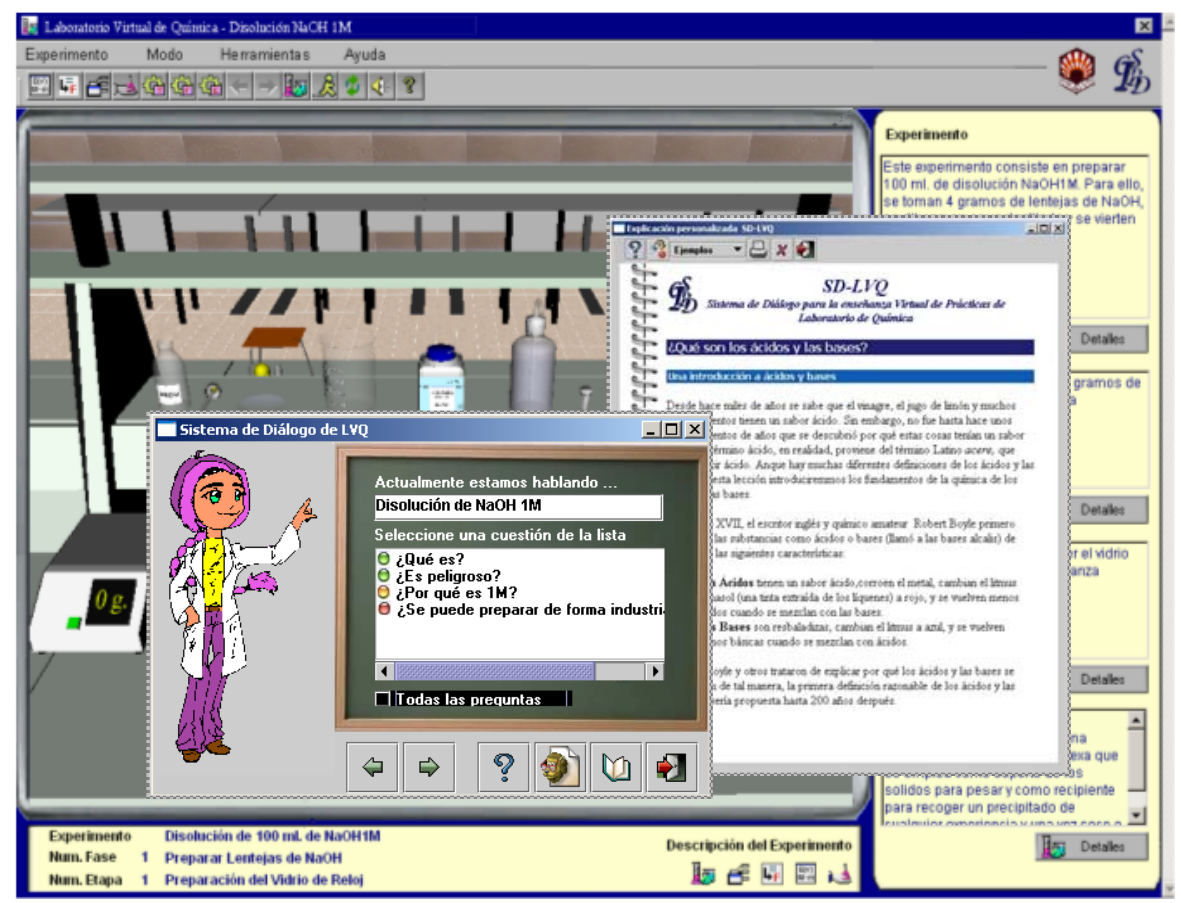

Fig. 4. A sample of the dialog system integrated in the Virtual Chemistry Lab

The initial user model is set up according to the user's experience and a series of indirect inferences based on the relationships between concepts or chunks held in the knowledge network, and the model is updated according to feedback from user interaction. Moreover, the student model also plays a part in the choice of dialog actions. The system uses the model to offer an explanation of a concept to the student, or perhaps to carry out a test.

\section{Discussion}

In this paper an ontology for the explanatory dialog generation is proposed. The representation of the knowledge to be explained is represented by a network of multiconnected chunks, where each chunk can be further specialized in specific knowledge frames, may contain associated explanations of varying degree of complexity and with different explanatory models, and can be associated with one or a set of questions the student might ask. Student model is represented through the belief of user's knowledge on chunks and throughout the learning process by the use of both stereotypes and direct or indirect inference, enabling the explanation to be adapted to the requirements of each student. 
The solution developed considers a student-dominated dialog in which the (virtual) teacher answers any pertinent questions posed by the student, providing a fair representation of the real teaching processes.

Based on the proposed model and employing the Java language [17] in conjunction with the Oracle 9i DBMS [18] for knowledge-base management, a system was developed to build the knowledge network. The teacher can define all the elements appearing in the dialog (chunks, questions, etc.) and interconnect them. These elements and their relationships are manipulated by the system through a drop-down hierarchical menu, enabling the student to easily browse the knowledge network.

While the predefined questions for each chunk are simple text sentences, explanations may be constructed as .html or .xml files (also stored in the nucleus of the database), thus allowing inclusion of multimedia content (text, graphics, images, and so on).

The system is flexible and may be incorporated into any other object-based teaching aid. This is achieved by importing the classes for which knowledge-explanation items are required and linking those classes to the knowledge (chunks, dialog questions, etc.), as we have carried out with Virtual Chemistry Laboratory [5] (see Figure $4)$.

\section{References}

1. Cartwright, H.M., Valentine, K. A Spectrometer in the Bedroom. The Development and Potential of Internet-Based Experiments. Computers \& Education. 2002, 38, 53-64.

2. Colwell, C., Scanlon, E., Cooper, M. Using Remote Laboratories to Extend Access to Science and Engineering. Computers \& Education, 2002, 38, 65-76.

3. Rzepa, H.S., Tonge, A.P., VchemLab: A Virtual Chemistry Laboratory. The Storage, Retrieval and Display of Chemical Information Using Standard Internet Tools, J. Chem. Inf. Comp. Sci., 1998, 36(6), 1048-1053.

4. (a) www.modelscience.com/products.html, (b) www2.acdlabs.com/ilabs,

(c) www. chemsw.com/10202.htm, (d) www.compuchem.com/dldref/formdem.htm,

(e) www.ir.chem.cmu.edu /irProject/applets/virtuallab/applet_wPI.asp,

(f) chem.lapeer.org/Chem1Docs/Index.html, (g) chem-www.mps.ohio-state.edu/ lars/ moviemol.html

5. Luque Ruiz, I. López Espinosa, E., Cerruela García, G. Gómez-Nieto, M.A. Design and Development of Computer-Aided Chemical Systems: Virtual Labs for Teaching Chemical Experiments in Undergraduate and Graduate Courses. J. Chem. Inf. Comput. Sci., 2001, 41(4), 1072-1082.

6. Crosier, J., Cobb, S., Wilson, J.R. Key Lessons for the Design and Integration of Virtual Environments in Secondary Science. Computers \& Education, 2002, 38, 77-94.

7. Moore, J.D. Participating in Explanatory Dialogues. MIT Pres, Cambridge, Massachusetts, 1995.

8. Cawsey, A.J. Explanation and Interaction: the Computer Generation of Explanatory Dialogues. MIT Pres, Cambridge, Massachusetts, 1992.

9. Sinclair J. McH., Coulthard R.M. Towards an Analysis of Discourse: The English Used by Teachers and Pupils. Londres. Oxford University Press. 1975. 
10. Nussbaum, M., Rosas, R., Peirano, I., Cárdenas, F. Development of Intelligent Tutoring Systems using Knowledge Structures. Computers \& Education. 2001, 36, 15-32.

11. Fernández-Castro, Y., Díaz Harraza, A., Verdejo, F. Architectural and Planning Issues in Intelligent Tutoring Systems. Journal of Artificial Intelligence and Education. 1993, 4 (4), 357-395.

12. Angelo C. Restificar, Syed S. Ali and Susan W. McRoy, ARGUER: Using Argument Schemas for Argument Detection and Rebuttal in Dialogs, In Proceedings of the Seventh International Conference on User Modelling (UM-99), Banff, Canada, June 20-24, 1999.

13. Soller, A. Computational Analysis of Knowledge Sharing in Collaborative Distance Learning Doctoral Dissertation. University of Pittsburgh, 2002.

14. Rumbaugh, J., Jacobson, I., Booch, G., The Unified Modeling Language. Reference Manual, Addison-Wesley Longman Inc, USA, 1999.

15. Ishizaki, M., Crocker, M., Mellish, C. Exploring Mixed-Initiative Dialogue using Computer Dialogue Simulation. User Modelling and User Interaction, 1999, 9(1), 79-91.

16. Rich, E. User Modeling Via Stereotypes. Cognitive Science, 1979, 3,339-354.

17. Campione, M. The Java Tutorial Third Edition. McGraw-Hill. 2002 (http://java.sun.com)

18. Loney, K., Koch, G.. Oracle 9i The Complete Reference. Oracle Press. 2002. 